Pacific Journal of Mathematic 


\title{
DISTRIBUTIVITY AND THE NORMAL COMPLETION OF BOOLEAN ALGEBRAS
}

\author{
R. S. PIERCE
}

1. Introduction. In a recent paper, [4], Smith and Tarski studied the interrelations between completeness and distributivity properties of a Boolean algebra. Independently, the author also obtained some of the results of Smith and Tarski. This work was reported in [2]. The present paper continues the study of distributivity in Boolean algebras. Specifically, it deals with the problem of imbedding a Boolean algebra $B$ in an $\alpha$-distributive, $\beta$-complete algebra, $\alpha$ and $\beta$ being infinite cardinal numbers. If it is required that the imbedding be regular, that is, preserve existing joins and meets, then (see [3]) the problem is equivalent to the question of when the normal completion of $B$ (or a subalgebra of the completion) is $\alpha$-distributive. Our two main results can be briefly stated as follows :

THEOREM 3.1. Every $\alpha$-distributive Boolean algebra can be regularly imbedded in an $\alpha$-complete, $\alpha$-distributive Boolean algebra.

Theorem 5.1. There exists an $\alpha$-field of sets whose normal completion is not $\alpha$-distributive.

Between these principal results, we obtain two simple conditions, one of which is necessary, the other sufficient for the normal completion of a Boolean algebra to be $\alpha$-distributive. These appear naturally as particular cases of more general facts relating properties which are similar to, but not identical with $\alpha$-distributivity and $\beta$-completeness.

2. Preliminary results. The notation of this paper will be the same as that of [2]. The Greek letters $\alpha, \beta$ and $\gamma$ always denote cardinal numbers, while $\rho, \sigma$ and $\tau$ are used as indices belonging to sets $R, S$ and $T$ respectively. The symbol $\infty$ will be used as though it were a largest cardinal. This is a notational convenience, and in no case involves questionable logic. As in [2], a subset $A$ of an arbitrary Boolean algebra $B$ is called a covering (of $B$ ) if the least upper bound of $A$ in $B$ is the unit $u$ of $B$. If the elements of the covering $A$ are disjoint, then $A$ is termed a partition. Finally, if the covering (partition) $A$ is of cardinality less than, or equal to $\alpha$, symbolically $\overline{\bar{A}} \leqq \alpha$, then $A$ is called an $\alpha$-covering (respectively, $\alpha$-partition). If $A$ and $\tilde{A}$ are subsets of $B$, then $\tilde{A}$ is said to refine $A$ when every $\tilde{a} \in \tilde{A}$ is $\leqq$ some $a \in A$.

Received December 28, 1956. In revised form July 11, 1957. 
Definition 2.1 (Smith-Tarski). A Boolean algebra $B$ is called ( $\alpha$, $\beta$ )-distributive if

$$
\wedge_{\sigma \in S} \vee_{\tau \in T} b_{\sigma \tau}=\vee_{\varphi \in F} \wedge_{\sigma \in S} b_{\sigma \varphi(\sigma)}, F=T^{S}
$$

holds identically when $\overline{\bar{S}} \leqq \alpha, \overline{\bar{T}} \leqq \beta$ and the bounds are assumed to exist in $B$.

Some elementary consequences of this definition are worth noting:

(2.2) If $B$ is $(\alpha, \beta)$-distributive and $\alpha^{\prime} \leqq \alpha, \beta^{\prime} \leqq \beta$, then $B$ is $\left(\alpha^{\prime}, \beta^{\prime}\right)$ distributive. Any regular ${ }^{1}$ subalgebra of an $(\alpha, \beta)$-distributive Boolean algebra is $(\alpha, \beta)$-distributive. Every Boolean algebra is $(n, \beta)$-distributive, where $n$ is finite and $\beta$ is arbitrary.

The last assertion of (2.2) is a variant of the Tarski-von Neumann theorem (see [1], p. 165). This infinite distributivity is a property of Boolean algebras which we use repeatedly and without mention.

A useful characterization of $(\alpha, \beta)$-distributive Boolean algebras is given by the following theorem, which, in somewhat different terms, appears in [4]. Since this characterization is used often in the sequal, we sketch a proof.

TheORem 2.3. Let $\alpha$ and $\beta$ be arbitrary cardinal numbers. A Boolean algebra $B$ is $(\alpha, \beta)$-distributive if and only if, for any family $\left\{A_{\sigma} \mid \sigma \in S\right\}$ of $\beta$ coverings of $B$ with $\overline{\bar{S}} \leqq \alpha$, there is a covering of $B$ which refines every $A_{\sigma}$.

Proof. Suppose $B$ is $(\alpha, \beta)$-distributive. Let $\left\{\mathrm{A}_{\sigma} \mid \sigma \in S\right\}$ be a given family of $\beta$-coverings with $\overline{\bar{S}} \leqq \alpha$. It can be assumed that every $\mathrm{A}_{\sigma}$ is indexed by the same set $T: A_{\sigma}=\left\{a_{\sigma \tau} \mid \tau \in T\right\}$. Let $\mathrm{A}=\{a \in B \mid\{a\}$ refines every $\left.A_{\sigma}\right\}$. Clearly $A$ refines every $A_{\sigma}$. If $A$ is not a covering of $B$, there exists $b \neq 0$ (the zero of $B$ ) which is disjoint from every $a \in A$. Setting $b_{\sigma \tau}=a_{\sigma \tau} \wedge b$, it is easy to see that $\wedge_{\sigma} \vee_{\tau} b_{\sigma \tau}=b>0=\vee_{\psi} \wedge_{\sigma} b_{\sigma \psi(\sigma)}$. This contradicts $(\alpha, \beta)$-distributivity. Thus $A$ is a covering.

Conversely, let $B$ satisfy the condition of the theorem. Suppose $\vee_{\tau \in T} b_{\sigma \tau}, \wedge_{\sigma \in S} \vee_{\tau \in T} b_{\sigma \tau}=b$ and $\wedge_{\sigma \in S} b_{\sigma \varphi(\sigma)}$ exist for all $\sigma \in S$ and all $\varphi \in F$ $=T^{s}$. Let $\omega$ be a symbol not in $T$. Put $T^{\prime}=T \cup\{\omega\}, b_{\sigma \omega}=b^{\prime}, \bar{A}_{\sigma}=\left\{b_{\sigma \tau} \mid\right.$ $\left.\tau \in T^{\prime}\right\}, b_{\varphi}=\wedge_{\sigma \in S} b_{\sigma \varphi(\sigma)}$ for all $\varphi \in F$. Then each $\bar{A}_{\sigma}$ is a $\beta$-covering, so by assumption there is a covering $A$ which refines every $\bar{A}_{\sigma}$. If $a \in A$, then either $a \leqq b_{\varphi}$ for some $\varphi \in F$, or else $a \leqq b^{\prime}$. Thus, if $c \geqq b_{\varphi}$ for all $\varphi$, $c \vee b^{\prime} \geqq$ l.u.b. $A=u$ (the unit of $B$ ). Hence, $c \geqq b$. Since $b$ is obviously an upper bound of all $b_{\varphi}$, it follows that $b=\wedge_{\varphi \in F} b_{\varphi}$.

For simplicity, an $(\alpha, \alpha)$-distributive B. A. is just called $\alpha$-distributive.

1 A subalgebra $\bar{B}$ of a Boolean algebra $B$ is called regular (see [3]) if, whenever $a$ $=$ l.u.b. $A$ in $\bar{B}(a \in \bar{B}, A \leqq \bar{B})$, then $a=1$.u.b. $A$ in $B$ also. Of course, in a Boolean algebra, this property implies its dual and conversely. 
Corollary 2.4. A Boolean algebra $B$ is $\alpha$-distributive if and only if every family $\left\{A_{\sigma} \mid \sigma \in S\right\}$ of binary partitions with $\overline{\bar{S}} \leqq \alpha$ has a common refining covering.

Indeed, if $\left\{\bar{A}_{\sigma} \mid \sigma \in S\right\} \quad(\overline{\bar{S}} \leqq \alpha)$ is a family of $\alpha$-coverings, say $\bar{A}_{\sigma}$ $=\left\{a_{\sigma \tau} \mid \tau \in T\right\}$, then, setting $A_{\sigma \tau}=\left[a_{\sigma \tau},\left(a_{\sigma \tau}\right)^{\prime}\right]$, the set $\left\{A_{\sigma \tau} \mid \sigma \in S, \tau \in T\right\}$ is a family of no more than $\alpha$ binary partitions of $B$ and any covering which refines all $A_{\sigma \tau}$ is a common refinement of all $\bar{A}_{\sigma}$ (because $\wedge_{\tau}\left(a_{\sigma \tau}\right)^{\prime}=0$.

For future reference, we list some of the well known properties of the normal completion (or completion by "cuts") of a Boolean algebra. The Stone-Glivenko theorem $((2.5)$ below) is proved in the standard reference [1]. The proofs of (2.6) to (2.8) are conveniently collected in [3].

(2.5) (Stone-Glivenko) The normal completion of a Boolean algebra is a Boolean algebra.

(2.6) Let $\bar{B}$ be the normal completion of the Boolean algebra $B$. Then $B$ is a regular subalgebra of $\bar{B}$.

(2.7) Any Boolean algebra $B$ is dense in its normal completion $\bar{B}$. That is, if $0 \neq \bar{b} \in \bar{B}$, then there exists $b \in B$ with $0 \neq \bar{b} \leqq b$.

(2.8) If the Boolean algebra $B$ is a dense subset of the complete Boolean algebra $\bar{B}$, then $\bar{B}$ is isomorphic to the normal completion of $B$. Moreover, if $B \subset \tilde{B} \subset \bar{B}$ and $\tilde{B}$ is complete, then $\tilde{B}=\bar{B}$.

Definition 2.9. Let $B$ be a Boolean algebra. Let $\bar{B}$ be the normal completion of $\mathrm{B}$. Let $\alpha$ be an infinite cardinal number. The normal $\alpha-$ completion of $B$ is the intersection of all $\alpha$-complete subalgebras of $\bar{B}$ which contain $B$. Denote this algebra $B^{\alpha}$. It will also be convenient to write $B^{\infty}$ for $\bar{B}$.

Clearly, $B^{\alpha}$ is the smallest $\alpha$-complete subalgebra of $B^{\infty}$ containing $B$. Moreover, $B$ is dense in $B^{\alpha}$ and is regularly imbedded in $B^{\alpha}$.

3. The imbedding theorem. The primary purpose of this section is to prove Theorem 3.1 (stated in the introduction). However, the method of the proof is used several times in the following sections, so it behooves us to present it in a form which is sufficiently general to cover all future needs.

Lemma 3.2. Let $\bar{B}$ be a complete Boolean algebra. Let $\mathfrak{A}$ be a nonempty family of partition of $\bar{B}$ such that if $\left\{A_{\sigma} \mid \sigma \in S\right\} \subseteq \mathfrak{U}$ and $\overline{\bar{S}} \leqq \alpha$, then some $A \in \mathfrak{A}$ refines every $A_{\sigma}$. Let $\tilde{B}$ be the set of all joins of subsets of the partitions $A$ in $\mathfrak{Y}$. Then $\tilde{B}$ is an $\alpha$-complete Boolean algebra such 
that $A \subseteq \tilde{B}$ for every $A \in \mathfrak{A}$ and every $\alpha$-covering of $\tilde{B}$ is refined by some $A \in \mathfrak{A}$. Hence, $\tilde{B}$ is $\alpha$-distributive.

Proof. If $C \subseteq A \in \mathfrak{A}$, then (l.u.b. $C)^{\prime}=$ l.u.b. $(A-C)$, since $A$ is a partition. Hence $\tilde{B}$ is closed under complementation. Suppose $\left\{c_{\sigma} \mid \sigma \in S\right\}$ is a subset of $\tilde{B}$ with $\overline{\bar{S}} \leqq \alpha$. By definition of $\tilde{B}$, for each $\sigma \in S$, there exists a partition $\mathrm{A}_{\sigma} \in \mathfrak{U}$ and a subset $C_{\sigma} \subseteq A_{\sigma}$ such that $c_{\sigma}=$ l.u.b. $C_{\sigma}$. Then $A_{\sigma}$ refines the binary partition $\left\{c_{\sigma},\left(c_{\sigma}\right)^{\prime}\right\}$. Let $A \in \mathfrak{A}$ be a common refinement of all $A_{\sigma}$. Then $A$ is a common refinement of all $\left\{c_{\sigma},\left(c_{\sigma}\right)^{\prime}\right\}$ and g.l.b. $\left\{c_{\sigma} \mid \sigma \in S\right\}=$ l.u.b. $\left\{a \in A \mid a \leqq c_{\sigma}\right.$ all $\left.\sigma \in S\right\} \in \tilde{B}$. Indeed, $c=$ l.u.b. $\left\{a \in A \mid a \leqq c_{\sigma}\right.$, all $\left.\sigma \in S\right\} \leqq \vee_{\sigma \in S} c_{\sigma}$ is clear. But also, $c^{\prime}=$ l.u.b. $\{a \in A \mid a \leqq$ $\left(c_{\sigma}\right)^{\prime}$, seme $\left.\sigma \in S\right\} \leqq \wedge_{\sigma \in S}\left(c_{\sigma}\right)^{\prime}=\left(\wedge_{\sigma \in S} c_{\sigma}\right)^{\prime}$. Hence, $\tilde{B}$ is an $\alpha$-complete B.A. Obviously, $A \subseteq \widetilde{B}$ for all $A \in \mathfrak{A}$. If $\tilde{A}$ is an $\alpha$-covering of $\widetilde{B}$, then, as proved above, every binary partition $\left\{c, c^{\prime}\right\}$ with $c \in \tilde{A}$ is refined by some $A_{c} \in \mathfrak{A}$. Choosing $A \in \mathfrak{A}$ to be a refinement of all these $A_{c}$ gives a refinement of $\tilde{A}$. In fact, any $a \in A$ satisfies either $a \leqq c$, or $a \leqq c^{\prime}$ for all $c \in \tilde{A}$. If $a \leqq c^{\prime}$ for every $c$, than $a \leqq \wedge_{c \in \tilde{A}} c^{\prime}=(\text { l.u.b. } \tilde{A})^{\prime}=0$, since $\tilde{A}$ is a covering. Thus every $a \in A$ satisfies $a \leqq c$ for some $c \in \tilde{A}$.

Proof of (3.1). Let $\bar{B}$ be the normal completion of $B$. Let 2 be the set of all partitions of $\bar{B}$, which are of the form $\Pi_{\sigma \in S} A_{\sigma}=\left\{b_{\varphi} \mid \varphi \in 2^{S}\right\}$, where the $A_{\sigma}=\left\{a_{\sigma 0}, a_{\sigma 1}\right\}$ are binary partitions of $B$ and $b_{\varphi}=\wedge_{\sigma \in S} a_{\sigma \varphi(\sigma)} \in \bar{B}$. The fact that $\Pi_{\sigma \in S} A_{\sigma}$ is a partition follows directly from the assumed distributivity of $B$. If $A_{\tau}=\Pi_{\sigma \in S(\tau)} A_{\sigma \tau} \in \mathfrak{A}$ for all $\tau \in T$ with $\overline{\bar{T}} \leqq \alpha$, then $A=\Pi_{\tau \in T} \Pi_{\sigma \in S(\tau)} A_{\sigma \tau} \in \mathfrak{A}$ is a common refinement of all $A_{\tau}$. Thus, the hypotheses of (3.2) are satisfied. Consequently, there is an $\alpha$-complete, $\alpha$-distributive Boolean algebra $\tilde{B}$ with $B \subseteq \tilde{B} \subseteq \bar{B}$. Since $B$ is a regular subalgebra of $\bar{B}$, it is also a regular subalgebra of $\tilde{B}$.

4. Conditions for distributivity. In this section, we will examine the following five properties of a Boolean algebra B:

( $\left.I_{\alpha}\right) \quad B$ is $\alpha$-complete;

( $\left.I I_{\alpha}\right)$ every subset of an $\alpha$-partition of $B$ has a l.u.b. in $B$;

$\left(I I I_{\beta}\right)$ every $\beta$-covering of $B$ can be refined by a $\beta$-partition;

$\left(I V_{\alpha \beta}\right) \quad B$ is $(\alpha, \beta)$-distributive ;

$\left(V_{\alpha \beta}\right)$ If $\left\{A_{\sigma} \mid \sigma \in S\right\}$ is a set of $\beta$-partitions of $B$ with $\overline{\bar{S}} \leqq \alpha$, then there is a covering of $B$ which is a common refinement of every $A_{\sigma}$.

Certain relations between these properties are more or less evident.

(4.1) (a) $I_{\alpha}$ and $I I_{\alpha}$ are hereditary in $\alpha$, that is, $I_{\alpha}$ implies $I_{\gamma}$ and $I I_{\alpha}$ implies $I I_{\gamma}$ for all $\gamma \leqq \alpha$;

(b) $I V_{\alpha \beta}$ and $V_{\alpha \beta}$ are hereditary in both $\alpha$ and $\beta$;

(c) $I_{\alpha}$ implies $I I_{\alpha}$;

(d) $I V_{\alpha \beta}$ implies $V_{\alpha \beta}$; 
(e) $V_{\alpha \beta}$ and $I I I_{\beta}$ together imply $I V_{\alpha \beta}$;

(f) if $I_{\alpha}$ holds for all $\alpha<\beta$, then $I I I_{\beta}$, is satisfied ;

(g) $I V_{\alpha \alpha}$ is equivalent to $V_{\alpha 2}$ and hence to $V_{\alpha \alpha}$;

(h) $I I I_{\infty}$ is always satisfied, so $I V_{\alpha \infty}$ is equivalent to $V_{\alpha \infty}$.

Proofs. The statements (a)-(e) are obvious. If $B$ is $\alpha$-complete for all $\alpha<\beta$, and $A=\left\{a_{\xi}\right\}$ is a $\beta$-covering of $B$ indexed by the set of all ordinals $\xi$ of cardinality less than $\beta$, then $\left\{c_{\xi} \mid \bar{\xi}<\beta\right\}$ will be a $\beta$-partition refining $A$ if $c_{\xi}=a_{\xi} \wedge\left(\vee_{\eta<\xi} a_{\eta}\right)^{\prime}$. The assertion of $(\mathrm{g})$ is a restatement of (2.4). Finally, with the help of Zorn's lemma, it is always possible to construct a partition to refine any covering. This construction, the details of which we omit, proves (h).

It appears from (4.1) (e)-(h) that the condition $V_{\alpha \beta}$ is only slightly weaker than $I V_{\alpha \beta}$. On the other hand, the condition $I I_{\alpha}$ is substantially weaker than $I_{\alpha}$, as the following example indicates. Let $X$ be a set of cardinality $\beta$; let $B$ be the Boolean algebra of finite subsets of $X$ and their complements. If $\alpha$ is any cardinal number less than $\beta$, then any $\alpha$-partition of $B$ is finite. Consequently, $B$ satisfies $I I_{\alpha}$. In one case however, the properties $I_{\alpha}$ and $I I_{\alpha}$ are equivalent, namely :

(4.2) $I I_{\infty}$ is equivalent to $I_{\infty}$.

Proof. Let $C$ be an arbitrary subset of $B$. Let $C^{\prime}=\{d \in B \mid d \wedge c=0$, all $c \in C\}$. Then clearly, $u$ is the only upper bound of the set $C u C^{\prime}$, that is, $C u C^{\prime}$ is a cover. By $(4.1)(\mathrm{h})$, there is a partition $A$ refining $C u C^{\prime}$. If $D=\{a \in A \mid\{a\}$ refines $C\}$, then $A-D=\{a \in A \mid a \wedge c=0$, all $c \in C\}$. Hence l.u.b. $C=$ l.u.b. $D$ exists by $I I_{\infty}$.

It is appropriate now to explain the object of studying the various properties listed above. Our main interest, of course, is the relation between $I_{\infty}$ and $I V_{\alpha a \alpha}$, and specifically we would like to find simple necessary and sufficient conditions for the normal completion of a Boolean algebra to satisfy $I V_{\alpha \alpha}$. It is rather easy to prove that $I V_{\alpha \infty}$ is sufficient and $I V_{\alpha \exp (\alpha)}$ is necessary for $\alpha$-distributivity in $B^{\infty}$. The effort to fit these two facts into a broader pattern leads to consideration of conditions $I I_{\beta}$ and $V_{\alpha \beta}$. It turns out that properties $I I_{\beta}$ and $V_{\alpha \beta}$ are tied together rather closely. Unfortunately $I_{\beta}$ and $I V_{\alpha \beta}$ do not enjoy such an intimate relationship and the two conditions mentioned above are the more or less accidental offspring of $I I_{\beta}$ and $V_{\alpha \beta}$ rather than the progeny of $I_{\beta}$ and $I V_{\alpha \beta}$.

THEOREM 4.3. If the Boolean algebra $B$ satisfies $V_{\alpha \beta}$ and $I I_{\gamma}$, where $\gamma=\beta^{\alpha}$, then $B$ satisfies $V_{a \gamma}$.

Proof. The theorem is trivial if $\alpha$ is finite, so it will be assumed 
that $\alpha$ is an infinite cardinal number. Let $A$ be a $\gamma$-partition of $B$. Then $A$ can be indexed by a subset of $T^{s}$, where $\overline{\bar{T}}=\beta$ and $\overline{\bar{S}}=\alpha$, say $A=\left\{a_{\varphi}\right\}$. Since $B$ satisfies $I I_{\gamma}$, it is meaningful to define $b_{\sigma \tau}=$ l.u.b. $\left\{a_{\varphi} \mid \varphi(\sigma)=\tau\right\}$ for each $\sigma \in S, \tau \in T$. Then $A_{\sigma}=\left\{b_{\sigma \tau} \mid \tau \in T\right\}$ is a $\beta$-partition of $B$ and it is easy to see that any common refinement of all $A_{\sigma}$ is also a refinement of $A$. Now suppose $\left\{A_{\rho} \mid \rho \in R\right\}$ is a set of $\gamma$-partitions of $B$ and $\overline{\bar{R}} \leqq \alpha$. For each $\rho$ in $R$, define (as above) a set of $\beta$-partitions $\left\{A_{\rho \sigma} \mid \sigma \in S_{\rho}\right\}$ with the property that a common refinement of every $A_{\rho \sigma}$ with $\sigma \in S_{\rho}$ is also a refinement of $A_{\rho}$. Consider the set of all $\beta$-partitions $\left\{A_{\rho \sigma} \mid \sigma \in S_{\rho}, \rho \in R\right\}$. There are at most $\alpha^{2}=\alpha$ of these, so by property $V_{\alpha \beta}$, there is a covering $A$ which refines every $A_{\rho \sigma}$. But then $A$ refines every $A_{\rho}$. Thus, $B$ satisfies $V_{\alpha \gamma}$.

Corollary 4.4 (Smith-Tarski [4]). If $B$ is $\alpha$-distributive and $2^{\alpha}-\mathrm{com}$ plete, then $B$ is $\left(\alpha, 2^{\alpha}\right)$-distributive.

CoRollary 4.5. A necessary condition that $B^{\beta}$ be $\alpha$-distributive, where $\beta \geqq 2^{\alpha}$, is that $B$ be $\left(\alpha, 2^{\alpha}\right)$-distributive.

Indeed, if $B^{\beta}$ is $\alpha$-distributive, then by (4.4) it is $\left(\alpha, 2^{\alpha}\right)$-distributive. But $B$ is a regular subalgebra of $B^{\beta}$ and hence (by (2.2)) $B$ is also $(\alpha$, $2^{\alpha}$ )-distributive.

We do not know whether the converse of 4.5 holds. That is, if $B$ is $\left(\alpha, 2^{x}\right)$-distributive, does it follow that $B^{2^{\alpha}}$ is $\alpha$-distributive? This seems doubtful, but if the goal of $2^{\alpha}$-completeness (that is, property $I_{2 \alpha}$ ) is replaced by the property $I I_{2} \alpha$, then a positive result is obtained (in Corollary 4.8 below).

Theorem 4.6. Let $B$ be an arbitrary Boolean algebra. Define $\bar{B}$ to be the intersection of all algebras $\tilde{B}$ with the property $I I_{\beta}$ such that $B \subseteq \tilde{B} \subseteq B^{\infty}$. Then $\bar{B}$ satisfies $I I_{\beta}$. Moreover, $\bar{B}$ has property $V_{\alpha \beta}$ if and only if $B$ has property $V_{\alpha \beta}$. Also, if $B$ is a-complete and satisfies $V_{a \beta}$, where $\beta^{\alpha}=\beta$, then $\bar{B}$ is $\alpha$-complete.

Proof. Clearly $\bar{B}$ satisfies $I I_{\beta}$. Since $B$ is a regular subalgebra of $\bar{B}$, the property $V_{\alpha \beta}$ for $\bar{B}$ implies the same property for $B$. To establish the converse, it is sufficient to show that every $\beta$-partition of $\bar{B}$ can be refined by a $\beta$-partition of $B$.

Let $\mathfrak{A}$ be the set of all $\beta$-partitions of $B$. By (2.5), every $A \in \mathfrak{U}$ can be considered as a partition of $B^{\infty}$. By (2.2), every finite subset of $\mathfrak{U}$ has a common refinement in $\mathfrak{A}$. Let $\widetilde{B}$ be the set of all joins in $B^{\infty}$ of a subset of some $A \in \mathfrak{Y}$. By (3.2), $\widetilde{B}$ is a Boolean algebra containing $B$. Clearly $\tilde{B} \subseteq \bar{B}$. Suppose $\tilde{A}$ is a $\beta$-partition of $\tilde{B}$, say $\tilde{A}=\left\{a_{\tau} \mid \tau \in T\right\}$. Then 
$a_{\tau}=\bigvee\left\{b_{\sigma \tau} \mid \sigma \in S_{\tau}\right\} \quad$ with $\quad b_{\sigma \tau} \in B, \quad b_{\sigma \tau} \wedge b_{\sigma^{\prime} \tau}=0$ for $\sigma \neq \sigma^{\prime}$, and $\overline{\bar{S}}_{\tau} \leqq \beta$. Consequently, $A=\left\{b_{\sigma \tau} \mid \sigma \in S_{\tau}, \tau \in T\right\}$ is a $\beta$-partition of $B$ which refines $\tilde{A}$. The join of any subset of $\tilde{A}$ is also the join of a subset of $A$ and therefore in $\tilde{B}$. Since $\tilde{A}$ was an arbitrary $\beta$-partition, $\tilde{B}$ has property $I I_{\beta}$. Consequently, $\bar{B} \leqq \tilde{B}$. Thus every $\beta$-partition of $\bar{B}=\tilde{B}$ can be refined by a $\beta$-partition of $B$.

Finally, suppose $B$ is $\alpha$-complete and satisfies $V_{\alpha \beta}$, with $\beta^{\alpha}=\beta$. If $\left\{A_{\sigma} \mid \sigma \in S\right\}, \overline{\bar{S}} \leqq \alpha$ is a set of $\beta$-partitions of $B$, then $\prod_{\sigma \in S} A_{\sigma}=\left\{\wedge_{\sigma \in S} b_{\sigma} \mid\right.$ $\left.b_{\sigma} \in A_{\sigma}\right\}$ is a $\beta^{\alpha}=\beta$-partition. Hence, by (3.2), $\widetilde{B}=\bar{B}$ is $\alpha$-complete.

Corollary 4.7. The normal completion of a Boolean algebra $B$ is $(\alpha, \infty)$-distributive if and only if $B$ is $(\alpha, \infty)$-distributive.

\section{Proof. By (4.6), (4.1) and (4.2).}

COROLlary 4.8. If the continuum hypothesis is true for the infinite cardinal $\alpha$ (that is, $2^{\alpha}$ covers $\alpha$ ), then an $\alpha$-complete Boolean algebra $B$ can be regularly imbedded in an $\alpha$-complete, $\alpha$-distributive algebra satisfying $I_{2^{\alpha}}$ if and only if $B$ is $\left(\alpha, 2^{\alpha}\right)$-distributive.

Proof. The sufficiency of $\left(\alpha, 2^{\alpha}\right)$-distributivity is a consequence of (4.6) and (4.1). The necessity follows from (4.3), (4.1) and (2.2).

5. An example. Because of (4.5), the Theorem (5.1) of the introduction can be proved by constructing an $\alpha$-field which is not $\left(\alpha, 2^{\alpha}\right)$ distributive.

Let $X$ be a set of cardinality $2^{\alpha}$. Denote by $Y$ the set of all ordinal numbers of cardinality less than $\alpha$. Let $Z$ be the set of all bounded functions in $Y^{x}$, that is, functions $f$ for which there is an $\eta \in Y$ such that $f(x)<\eta$ for all $x$ in $X$. Let $\mathscr{L}$ be the collection of all sets of the form

$$
L=L_{W, \varphi}=\left\{f \in Z|f|_{W}=\varphi\right\},
$$

where $W \leqq X, \bar{W} \leqq \alpha$ and $\varphi \in Y^{W}$. It is obvious that $\mathscr{L}$ contains the empty set and is closed under $\alpha$-intersections.

Let $\mathscr{F}$ be the $\alpha$-field generated by $\mathscr{C}$. It is to be shown that $\mathscr{F}$ is not $\left(\alpha, 2^{\alpha}\right)$-distributive. The proof hinges on a lemma, which is useful in its own right.

Lemma 5.2. Let $Z$ be a set. Suppose $\mathscr{L}$ is a nonempty family of subsets of $Z$ with the following properties:

(i) every $\alpha$-intersection of sets in $\mathscr{L}$ is in $\mathscr{L}$;

(ii) the complement of any set of $\mathscr{L}$ is a union of sets of $\mathscr{L}$. Let $\mathscr{F}$ be the $\alpha$-field generated by $\mathscr{L}$. Then $\mathscr{L}$ is dense in $\mathscr{F}$. 
Proof. Let $\overline{\mathfrak{B}}$ be the complete B. A. of all subsets of $Z$. Let $\mathfrak{A}$ be the collection of all partitions $A$ of $\overline{\mathfrak{B}}$ with $A \subseteq \mathscr{L}$. If $\left\{A_{\sigma} \mid \sigma \in S\right\} \cong \mathfrak{A}$, $\overline{\bar{S}} \leqq \alpha$ and say $A_{\sigma}=\left\{L_{\sigma \tau} \mid \tau \in T_{\sigma}\right\}$, then by (i), $\prod_{\sigma \in S} A_{\sigma}=\left\{\cap_{\sigma \in S} L_{\sigma \varphi(\sigma)} \mid \varphi\right.$ $\left.\in \prod_{\sigma \in S} T_{\sigma}\right\}$ is in $\mathfrak{A}$ and refines every $A_{\sigma}$. Let $\tilde{\mathfrak{B}}$ consist of all sets $V \subseteq Z$ such that both $V$ and $V^{c}$ are disjoint unions of set of $\mathscr{L}$. By (3.2) and (ii), $\mathscr{\mathscr { C }} \subseteq \tilde{\mathfrak{B}}+\overline{\mathfrak{B}}$ and $\tilde{\mathfrak{B}}$ is an $\alpha$-field. Thus, $\mathscr{L} \subseteq \mathscr{F} \cong \tilde{\mathfrak{B}}$. Since every set of $\tilde{\mathfrak{B}}$ is a union of sets of $\mathscr{L}$, the same is true of $\mathscr{F}^{-}$and in particular, $\mathscr{L}$ is dense in $\mathscr{F}$.

We now proceed to prove that $\mathscr{F}$ is not $\left(\alpha, 2^{\alpha}\right)$-distributive. For each pair $(x, \eta)$ with $x \in X$ and $\eta \in Y$, define $T_{(x, \eta)}=\{f \in Z \mid f(x)=\eta\}$. Clearly $T_{(x, \eta)} \in \mathscr{L}$. For each $\eta \in Y$, let $A_{\eta}=\left\{T_{(x, \eta)} \mid x \in X\right\}$. The argument is completed by showing

(1) $A_{\eta}$ is a $2^{\alpha}$-covering of $\mathscr{F}$;

(2) no covering of $\mathscr{F}$ refines every $A_{\eta}$.

Proof of (1). Evidently, $\overline{\bar{A}}_{\eta}=2^{\alpha}$, so the only thing to prove is that the l.u.b. of $A_{\eta}$ in $\mathscr{F}$ is $Z$. The first step is to show that the conditions (i) and (ii) of (5.2) are fulfilled, so that $\mathscr{L}$ is dense in $\mathscr{F}$. Condition (i) is clear. For condition (ii), let $L=L_{W, \varphi} \in \mathscr{L}$. Then $L^{c}=\cup_{x \in W}\{f \in Z \mid$ $f(x) \neq \varphi(x)\}=\cup_{x \in W}\left(\cup\left\{T_{(x, \eta)} \mid \eta \neq \varphi(x)\right\}\right)$ is a union of sets of $\mathscr{L}$.

Since $\mathscr{L}$ is dense in $\mathscr{F}$, it is enough, in proving (1), to show that if $L \in \mathscr{L}$ satisfies $L \cap T_{(x, \eta)}=\phi$ for all $x$, then $L=\phi$. Suppose $L \neq \phi$ and say $L=L_{W, \varphi}$. Pick $f \in L$ and let $x \in X-W$. Define $g \in Z$ by $g(x)=\eta$, $g(y)=f(y)$ if $y \neq x$. Then $g \in T_{(x, \eta)}$ and $g \in L$. Hence, $L \cap T_{(x, \eta)} \neq \phi$, which is the required conclusion.

Proof of (2). First note that $\cap_{\eta \in Y}\left(\cup A_{\eta}\right)=\phi$. For otherwise there would be an $f \in Z$ whose range included every $\eta \in Y$, contray to the boundedness of the functions of $Z$. But if $A$ is a subset of $\mathscr{F}$ which refines every $A_{\eta}$, then $\cup A \subseteq \cup A_{\eta}$ for all $\eta$. Hence, $\cup A \subseteq \cap_{\eta \in Y}\left(\cup A_{\eta}\right)=\phi$, so $A$ cannot be a covering.

\section{REFERENCES}

1. G. Birkhoff, Lattice theory, revised edition, 1948.

2. R. S. Pierce, Distributivity in Boolean algebras, Pacific J. Math. 5 (1957).

3. R. Sikorski, Products in Boolean algebras, Fund. Math. 37 (1950), 25-54.

4. E. C. Smith and A. Tarski, Higher degrees of completeness and distributivity in Boolean algebras, Trans. Amer. Math. Soc. 84 (1957), 230-257.

UNIVERSITY OF WASHINGTON 


\section{PACIFIC JOURNAL OF MATHEMATICS}

\section{EDITORS}

David Gilbarg

Stanford University

Stanford, California

R. A. Beaumont

University of Washington

Seattle 5 , Washington
A. L. Whiteman

University of Southern California Los Angeles 7, California

E. G. Straus

University of California

Los Angeles 24, California

\section{ASSOCIATE EDITORS}
E. F. BECKENBACH
C. E. BURGESS
M. HALL
E. HEWITT
A. HORN
V. GANAPATHY IYER
R. D. JAMES
M. S. KNEBELMAN

L. NACHBIN

I. NIVEN

T. G. OSTROM

H. L. ROYDEN
M. M. SCHIFFEI

G. SZEKERES

F. WOLF

K. YOSIDA

\section{SUPPORTING INSTITUTIONS}

UNIVERSITY OF BRITISH COLUMBIA

CALIFORNIA INSTITUTE OF TECHNOLOGY

UNIVERSITY OF CALIFORNIA

MONTANA STATE UNIVERSITY

UNIVERSITY OF NEVADA

OREGON STATE COLLEGE

UNIVERSITY OF OREGON

OSAKA UNIVERSITY

UNIVERSITY OF SOUTHERN CALIFORNIA
STANFORD UNIVERSITY

UNIVERSITY OF TOKYO

UNIVERSITY OF UTAH

WASHINGTON STATE COLLEGE

UNIVERSITY OF WASHINGTON

AMERICAN MATHEMATICAL SOCIETY

CALIFORNIA RESEARCH CORPORATION

HUGHES AIRCRAFT COMPANY

THE RAMO-WOOLDRIDGE CORPORATION

Printed in Japan by Kokusai Bunken Insatsusha

(International Academic Printing Co., Ltd.), Tokyo, Japan 


\section{Pacific Journal of Mathematics}

\section{Vol. 8, No. 1 \\ March, 1958}

Shimshon A. Amitsur, Commutative linear differential operators ......... 1

Masahiko Atsuji, Uniform continuity of continuous functions of metric

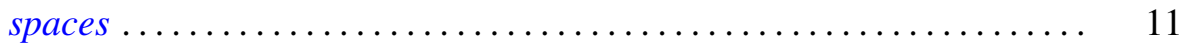

S. P. Avann, A numerical condition for modularity of a lattice . . . . . . . . . 17

Raymond G. D. Ayoub, A mean value theorem for quadratic fields........ 23

Errett Albert Bishop, Subalgebras of functions on a Riemann surface ..... . 29

Shaul Foguel, The relations between a spectral operator and its scalar

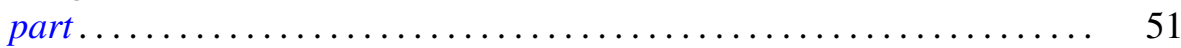

John Rolfe Isbell, Euclidean and weak uniformities ................. 67

Samuel Karlin and James L. McGregor, Many server queueing processes with Poisson input and exponential service times .............. 87

Paul Joseph Kelly and Ernst Gabor Straus, Curvature in Hilbert geometries....................................... 119

John W. Lamperti, Stationary measures for certain stochastic processes . . . 127

Richard Scott Pierce, Distributivity and the normal completion of Boolean

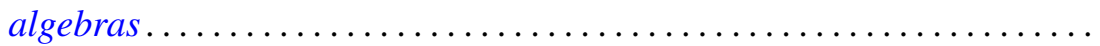

F. M. Ragab, Transcendental addition theorems for the hypergeometric

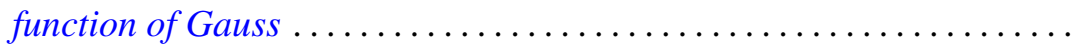

William T. Reid, Principal solutions of non-oscillatory self-adjoint linear differential systems ................................ 147

Maurice Sion, On general minimax theorems .................... 171

Chien Wenjen, On semi-normed ${ }^{*}$-algebras .................... 177 